\title{
Matéria
}

Revista Matéria, v. 11, n. 3, pp. 197 - 203, 2006

ISSN 1517-7076

http://www.materia.coppe.ufrj.br/sarra/artigos/artigo10790

\section{Comportamento Mecânico e Características Estruturais de Compósitos Poliméricos Reforçados com Fibras Contínuas e Alinhadas de Curauá}

\author{
Sergio N. Monteiro ${ }^{\mathrm{a}}$, Regina Coeli M. P. Aquino ${ }^{\mathrm{b}}$, Felipe P. D. Lopes ${ }^{\mathrm{a}}$, Eduardo A. de Carvalho ${ }^{\mathrm{a}}$, José \\ Roberto M. d'Almeida ${ }^{\mathrm{C}}$ \\ ${ }^{a}$ Universidade Estadual do Norte Fluminense, UENF, Laboratório de Materiais Avançados, LAMAV, Av. \\ Alberto Lamego, 2000, 28013-602, Campos dos Goytacazes, Brasil \\ e-mail: sergio.neves@ig.com.br, felipeperisse@pop.com.br, eatem@uenf.br. \\ ${ }^{\mathrm{b}}$ Centro Federal de Educação Tecnológica, CEFET-Campos, Campos dos Goytacazes, RJ , Brasil. \\ e-mail: araquino4@oi.com.br \\ c Departamento de Ciência dos Materiais e Metalurgia, Pontifícia Universidade Católica do Rio de Janeiro, \\ PUC-Rio, Rio de Janeiro, Brasil. \\ e-mail: dalmeida@dcmm.puc-rio.br
}

RESUMO

Compósitos de fibras naturais lignocelulósicas reforçando matriz polimérica já estão sendo utilizados em diversos campos de interesse aplicado. Isto tem incentivado pesquisas científicas e tecnológicas tanto em fibras tradicionais, como o sisal e a juta, quanto naquelas, como as do curauá, que apresentam características promissoras. Até hoje, entretanto, os estudos sobre o comportamento de compósitos poliméricos de curauá utilizaram fibras curtas, descontínuas e aleatoriamente orientadas. Em conseqüência, a resistência mecânica obtida foi relativamente baixa. No presente trabalho investigou-se as propriedades de compósitos de matriz poliéster reforçada com até 30\% em peso de fibras contínuas e alinhadas de curauá. Estes compósitos foram ensaiados em flexão e a superfície de fratura observada por microscopia eletrônica de varredura. Os resultados mostram resistência superior à obtida por outros pesquisadores em compósitos com fibras curtas e não-orientadas. Observações microestruturais revelaram efetiva adesão das fibras à matriz, o que contribuiu para o desempenho mecânico dos compósitos.

Palavras chave: Compósitos, fibras de curauá, resistência à flexão, microestrutura.

\section{Mechanical Behavior and Structural Characteristics of Polymeric Composites Reinforced with Continuous and Aligned Curaua Fibers}

\section{ABSTRACT}

Polymeric matrix composites reinforced with natural lignocellulosic fibers are now being used in many fields of practical interest. This has motivated scientific and technological investigations on both, traditional fibers, such as sisal and jute, as well as those, like curaua, which present promising characteristics. Until today, however, the works performed on the behavior of curaua reinforced polymeric composites used short, discontinuous and randomly oriented fibers. As a consequence the attained mechanical strength was relatively small. In the current work the properties of polyester matrix composites reinforced with up to 30 wt. \% of continuous and aligned curaua fibers was investigated. These composites were bend tested and the fracture surface was observed by scanning electron microscopy. The results showed strength values higher than those obtained by other researchers in curaua composites with short and non-oriented fibers. Microstructural observations revealed an effective adhesion between the fibers and the matrix, which contributed to the mechanical performance of the present composites.

Keywords: Composites, curaua fibers, flexural strength, microstructure.

\section{INTRODUÇÃO}

O curauá, cujo nome científico, Ananas erectifolius, o identifica como sendo da família do abacaxi, (Ananas comosus), é uma planta da região amazônica com grande potencial para o uso de suas fibras 
lignocelulósicas [1, 2]. Embora seu fruto seja comestível, o interesse econômico pelo curauá está primordialmente associado às fibras extraídas de suas folhas como mostrado na Figura 1. Estas folhas são rígidas eretas e com faces planas, podendo alcançar mais de um metro de comprimento e quatro centímetros de largura [2]. As fibras extraídas são relativamente macias e com elevada resistência mecânica, comparativamente com outras fibras lignocelulósicas. Segundo Leão et. all. [2]], as fibras de curauá estão entre as quatro lignocelulósicas mais rígidas até hoje conhecidas. Originalmente na Amazônia, as fibras de curauá eram utilizadas pelos índios para tecer redes de dormir, fazer cordas e linhas de pesca. Atualmente fibras picadas de curauá estão sendo misturadas com sobras de cobertores e tapetes descartadas pela industria têxtil para reforçar matriz de polipropileno em compósitos utilizados no teto e na parte interna do compartimento de bagagem de automóveis como o Fox e o Pólo da Wolkswagen do Brasil [3]].

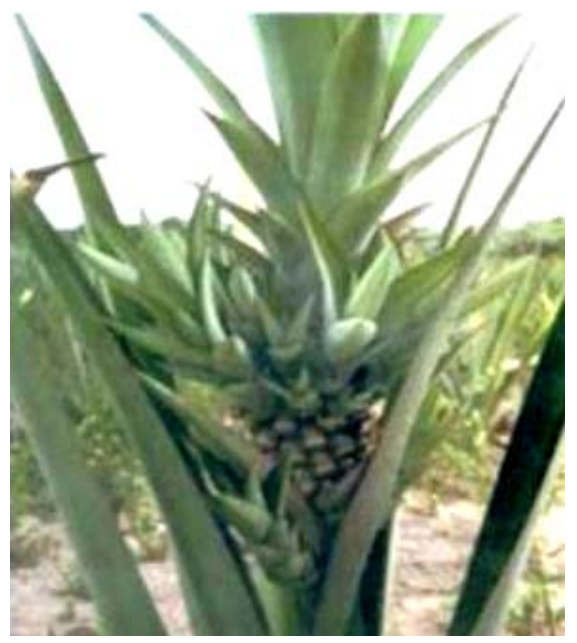

Figura 1: Planta de curauá mostrando fruto e folhas.

Pesquisas também vêm sendo realizadas [4-7] para avaliar as propriedades de compósitos poliméricos reforçados com fibras de curauá. Os resultados obtidos até agora são bastante animadores, uma vez que as fibras de curauá apresentam resistência mecânica [4] comparável à das fibras de sisal e juta [8]. Além disso, compósitos reforçados com $20 \%$ em peso de fibras de curauá picadas em matriz de poliuretano e polipropileno apresentam um substancial aumento na rigidez $[\underline{5}, \underline{6}]$. Mais recentemente foi mostrado que a microestrutura de cada fibra de curauá, constituída de um feixe de filamentos, pode ajudar na aderência da resina polimérica []].

É importante mencionar que as investigações sobre compósitos de curauá realizadas até agora [2, $\underline{5}$, 6] só utilizaram material picado, ou seja, o reforço à matriz polimérica é considerado como sendo de fibras curtas, descontínuas e aleatoriamente orientadas. Em consequiência, a resistência mecânica destes compósitos com até $50 \%$ de fibra em matriz de polipropileno, mesmo utilizando agente acoplante, não ultrapassou 33 MPa.

Em princípio, fibras longas e alinhadas são as que propiciam maior resistência aos compósitos [9, 10]. Assim, o objetivo do presente trabalho foi investigar as propriedades de compósitos de matriz poliéster reforçada com até 30\% em peso de fibras contínuas e alinhadas de curauá sem qualquer tratamento e sem adição de produtos químicos que aumentassem o acoplamento fibra/matriz. A idéia foi de reduzir ao máximo possível o custo de processamento dos compósitos, tornando-os assim economicamente mais competitivos.

\section{MATERIAIS E MÉTODOS}

Fibras longas de curauá foram adquiridas da firma Fibras Naturais S.A. de São Paulo que comercializa o produto vindo diretamente do Estado do Pará. A Figura 2 ilustra o aspecto das fibras de curauá utilizadas na pesquisa. 


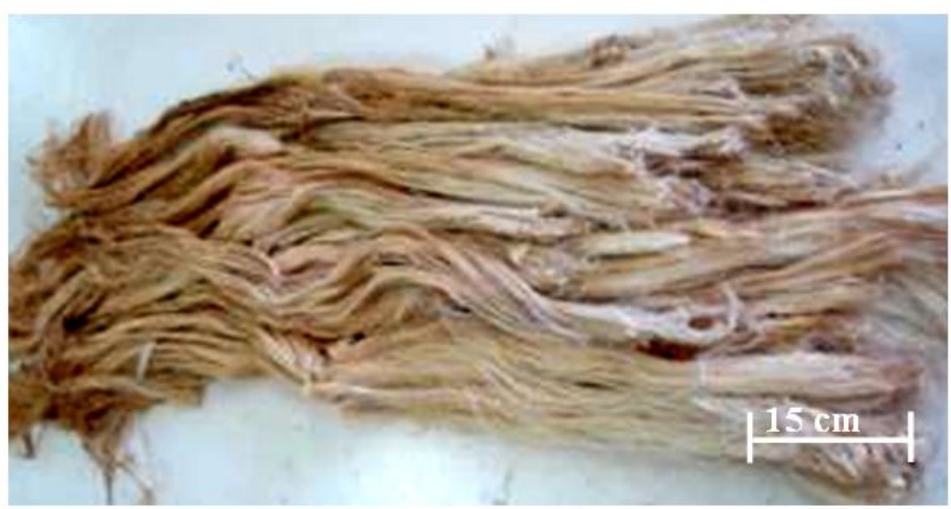

Figura 2: Fibras longas de curauá como comercializadas.

Estas fibras foram utilizadas na condição de como recebidas sem qualquer tratamento superficial, exceto uma rápida limpeza e secagem ao ar livre.

O comprimento e o diâmetro das fibras foram estatisticamente avaliados utilizando-se os histogramas de medidas da Figura 3.

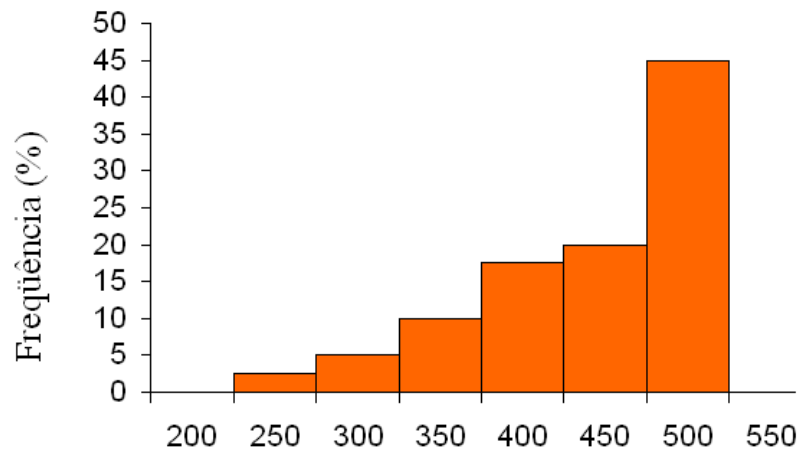

Comprimento (mm)

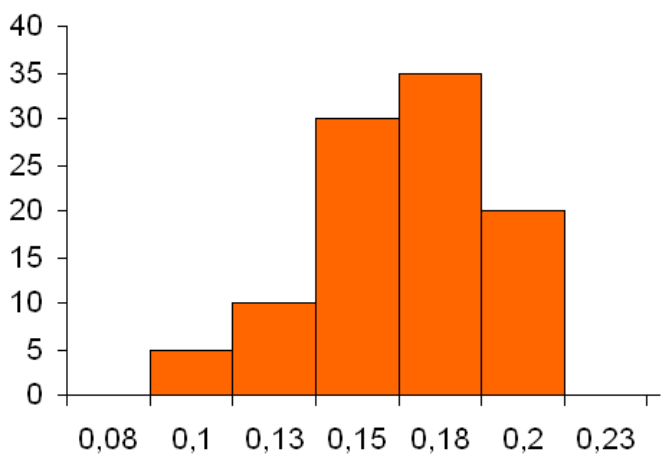

Diâmetro (mm)

Figura 3: Distribuição de medidas de comprimento e diâmetro das fibras de curauá.

A partir dos gráficos da Figura 3 calcularam-se o comprimento médio, $\mathrm{L}=442 \mathrm{~mm}$, e o diâmetro médio, $\mathrm{d}=0,17 \mathrm{~mm}$.

Compósitos de matriz polimérica reforçados com 5, 10, 15, 20, 25 e 30\% em peso de fibras foram confeccionados sob pressão de $0,53 \mathrm{MPa}$ à temperatura ambiente, da ordem de $25^{\circ} \mathrm{C}$, e curados por 24 horas. A resina polimérica utilizada como matriz foi um poliéster tipo ortoftálico endurecido com $0,5 \%$ de catalisador à base de metil-etil cetona.

Placas retangulares destes compósitos medindo 152 x 122 x $1 \mathrm{~mm}$ foram fabricadas em um molde fechado de aço. Neste molde as fibras de curauá foram colocadas, na quantidade desejada, paralelamente e em toda a extensão da largura de $122 \mathrm{~mm}$ do mesmo. A quantidade complementar de resina poliéster misturada com o catalisador, ainda no estado líquido, foi misturada com as fibras. Durante a cura, aplicou-se carga de uma tonelada sobre a tampa do molde para facilitar a impregnação da resina por entre as fibras. Cada placa, após cura de um dia, foi então cortada, ao longo da direção das fibras em 6 corpos-de-prova com dimensões de 122 x 25 x $10 \mathrm{~mm}$.

Os corpos-de-prova foram ensaiados por flexão, usando o teste padronizado de 3 pontos em máquina Instron modelo 5582 com capacidade de $100 \mathrm{kV}$ a uma taxa de deformação de 1,6×10-2 $\mathrm{s}^{-1}$.

Do valor da carga máxima $Q_{m}$ obtida em cada ensaio, calculou-se a tensão máxima associada à resistência do compósito através da equação: 


$$
\sigma_{m}=\frac{3 L Q_{m}}{2 b d^{2}}
$$

sendo L a distância entre suportes igual a $90 \mathrm{~mm}$, b a largura igual a $25 \mathrm{~mm}$ e d a espessura igual a $10 \mathrm{~mm}$. Tendo em vista estes valores, a relação de abertura para profundidade ("span-to-depth ratio") foi sempre de 9, o que está dentro do intervalo exigido por norma para este tipo de ensaio [11]. Com os valores constantes dos corpos-de-prova e da condição de ensaio de flexão, a tensão foi obtida diretamente da relação:

$$
\sigma_{\mathrm{m}}(\mathrm{MPa})=54 \times 10^{-3} Q_{m}(\mathrm{~N})
$$

Fibras de curauá, empregadas em ensaios de pullout para avaliação do comprimento crítico relativamente à matriz de poliéster [12], foram analisadas por microscopia eletrônica de varredura, MEV. Amostras destas fibras foram montadas em suporte de alumínio com fita de carbono e então metalizadas com ouro para observação de imagens geradas por elétrons secundários, acelerados entre 15 e $25 \mathrm{kV}$, em um microscópio Jeol modelo JSM - 6460 LV do Programa de Engenharia Metalúrgica e de Materiais, PEMM, da COPPE/UFRJ.

\section{RESULTADOS E DISCUSSÃO}

A Figura 4 ilustra o aspecto das curvas de carga vs. deslocamento obtidas nos ensaios de flexão de três pontos para corpos-de-prova com diferentes frações de fibra de curauá. Nos gráficos desta figura observa-se que algumas curvas são contínuas, como a do corpo-de-prova com $30 \%$ de curauá enquanto a do corpo-de-prova com $5 \%$ de curauá apresenta descontinuidades logo após a carga máxima. As descontinuidades aparentemente correspondem à súbita ruptura de filamentos que constituem as fibras como será discutido mais adiante.

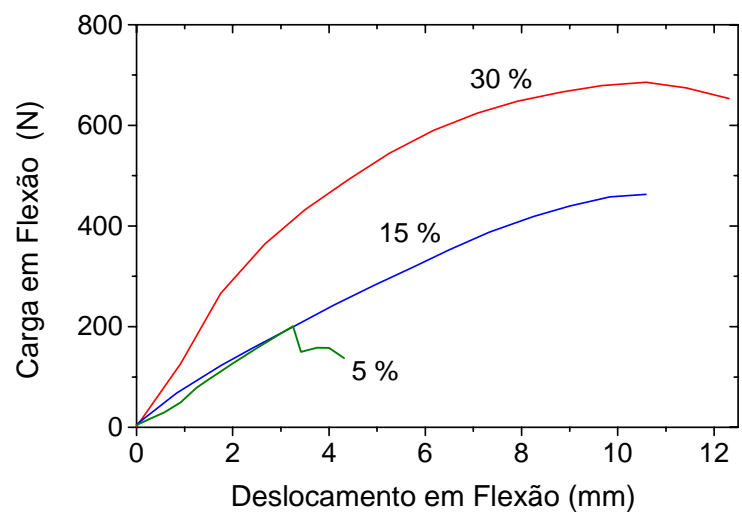

Figura 4: Micrografia por MEV das fibras trançadas de juta formando os fios do tecido.

Tabela 1: Tensão máxima dos compósitos de fibra de curauá reforçando resina poliéster.

\begin{tabular}{cc}
\hline Fração em peso da fibra de curauá (\%) & Tensão máxima em flexão (MPa) \\
\hline 0 & $41,4 \pm 6,9$ \\
5 & $47,0 \pm 15,2$ \\
10 & $80,0 \pm 20,3$ \\
15 & $80,1 \pm 23,1$ \\
20 & $96,6 \pm 13,0$ \\
25 & $91,0 \pm 23,6$ \\
30 & $94,0 \pm 16,5$ \\
\hline
\end{tabular}

A partir dos resultados de curvas como as da Figura 4 obteve-se o valor das cargas máximas e calculou-se, através da Equação 2, a tensão máxima para os diversos compósitos investigados.

A Tabela 1 apresenta os resultados das médias da tensão máxima em flexão de 6 corpos-de-prova para cada fração de fibra de curauá investigada. Nesta tabela também estão apresentados os correspondentes desvios padrão para cada valor de $\sigma_{\mathrm{m}}$. 
Com base nos dados da Tabela 1 construiu-se a curva de variação da resistência máxima à flexão dos compósitos em função da fração em peso de fibras de curauá como está representado na Figura 5.

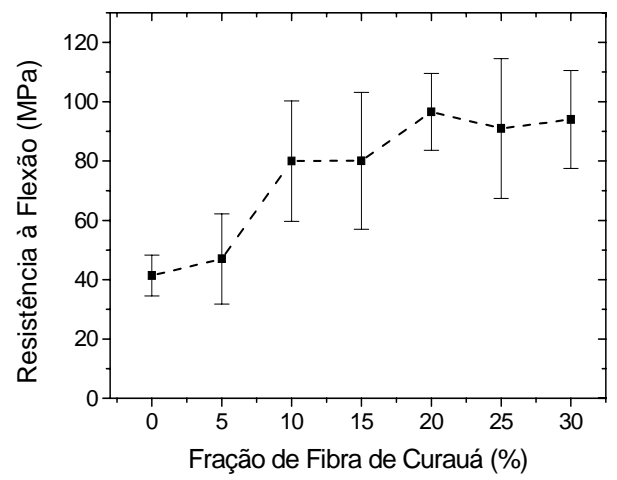

Figura 5: Curva de variação da resistência máxima à flexão dos compósitos de matriz poliéster com a fração de fibra de curauá.

Na curva da Figura 5 observa-se um significativo aumento na resistência com introdução de fibras longas de curauá na matriz de poliéster. Acima de 15\% de fibra nota-se uma tendência de nivelamento do valor da resistência pouco abaixo de $100 \mathrm{MPa}$. Na verdade, individualmente, um dos corpos de prova atingiu resistência à flexão de $125 \mathrm{MPa}$. Estes valores são praticamente o triplo dos maiores valores até agora obtidos em compósitos com fibras curtas [2]. Deve-se ainda mencionar que a quantidade de fibras curtas daquele trabalho chegava a $50 \%$.

A razão para a resistência mecânica superior das fibras longas em comparação com as curtas está relacionada à maior capacidade das primeiras transferirem os esforços aplicados sobre a matriz diretamente para as fibras $[\underline{9}, \underline{10}]$. Existem, entretanto, aspectos microestruturais da interação entre fibra de curauá e resina poliéster que valem a pena ser analisados.

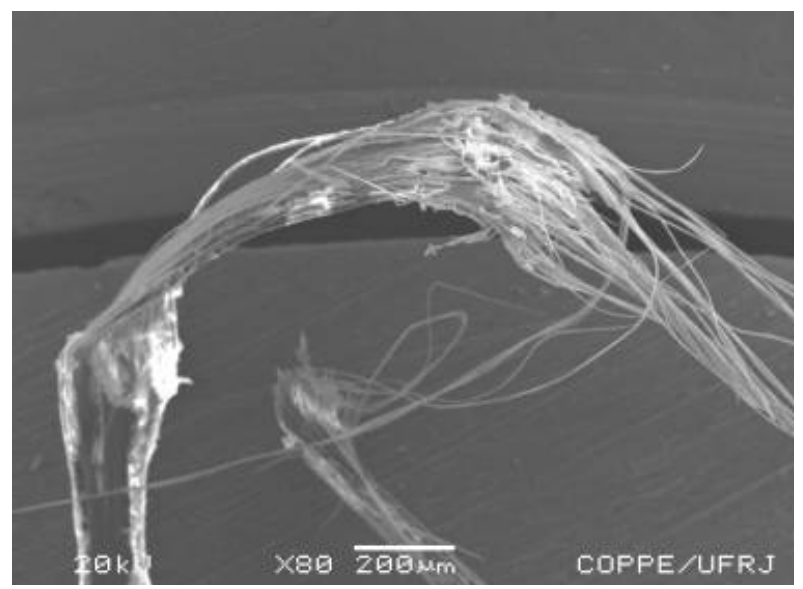

Figura 6: Fratura em tração de uma fibra de curauá.

A Figura 6 apresenta uma micrografia por MEV da ponta da fratura em tração de uma fibra de curauá. Nesta figura observa-se que a fibra de curauá é formada por várias fibrilas, como normalmente acontece em fibras lignocelulósicas. No caso do curauá algumas fibrilas, durante solicitação mecânica, podem se romper enquanto outras permanecem intactas. Isto claramente indica que uma única fibra de curauá age, por si, como um mini-compósito natural com relativamente longas e contínuas fibrilas capazes, cada uma, de absorver esforços da matriz.

A Figura 7 mostra uma fibra de curauá, parcialmente impregnada com resina poliéster, após descolar-se da matriz. 


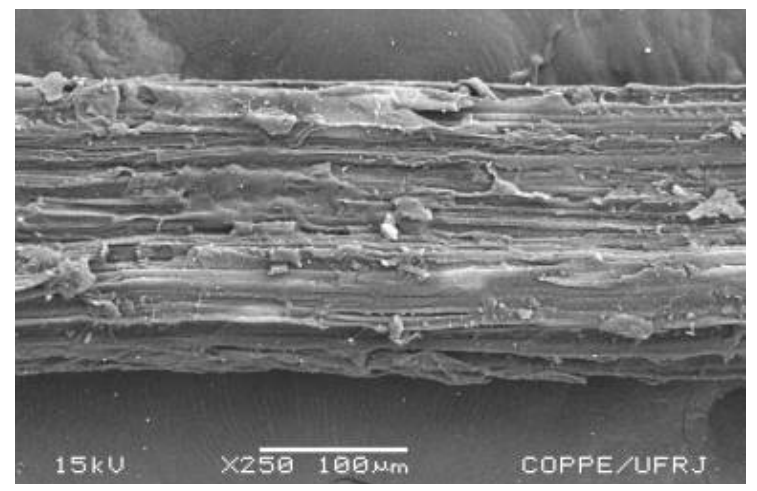

Figura 7: Fibra de curauá após descolamento da matriz de poliéster.

Nesta figura fica evidente a boa aderência da resina à fibra acompanhando o feixe de fibrilas. A dificuldade em descolar as fibrilas superficiais da cobertura de poliéster se traduz em eficiente adesão e capacidade de serem transferidos esforços mecânicos da matriz para toda a fibra.

A Figura 8, com maior aumento, ilustra o feixe de fibrilas revelado por baixo de uma camada aderida de poliéster. Nesta figura é relevante destacar a topografia do feixe de fibrilas que permite a penetração da resina de poliéster, quando ainda líquida durante o processamento, por entre as próprias fibrilas. Assim, além do efeito das fibras contínuas e alinhadas de curauá com diâmetro médio de 0,17 mm, Figura 3, pode-se também imaginar a formação de mini-compósitos com as próprias fibrilas, com diâmetro médio de $5 \mu \mathrm{m}$, reforçando a superfície da fibra à qual pertencem.

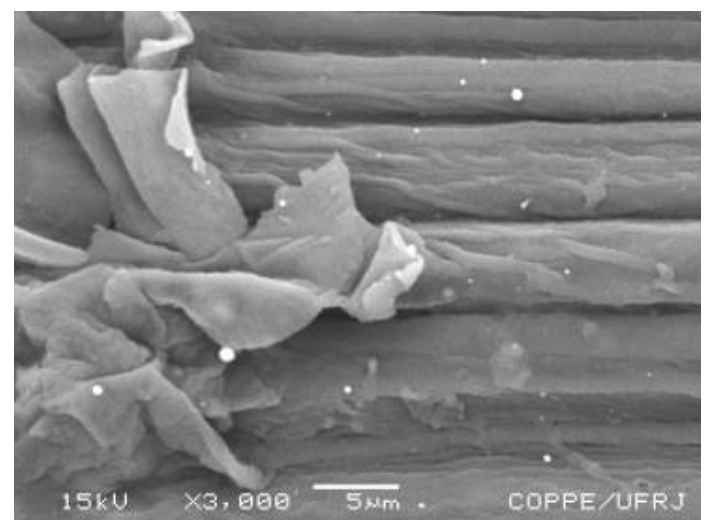

Figura 8: Feixe de fibrilas de uma fibra de curauá.

Nesta figura é relevante destacar a topografia do feixe de fibrilas que permite a penetração da resina de poliéster, quando ainda líquida durante o processamento, por entre as próprias fibrilas. Assim, além do efeito das fibras contínuas e alinhadas de curauá com diâmetro médio de $0,17 \mathrm{~mm}$, Figura 3, pode-se também imaginar a formação de mini-compósitos com as próprias fibrilas, com diâmetro médio de $5 \mu \mathrm{m}$, reforçando a superfície da fibra à qual pertencem.

Uma vez que estas fibrilas podem resistir individualmente à solicitação mecânica, mesmo que outras já tenham se rompido, Figura 7, a capacidade de o conjunto sustentar esforços é bastante eficaz. Esta é, provavelmente, a razão dos compósitos investigados no presente trabalho possuírem níveis de resistência, Tabela 1 e Figura 5, comparáveis aos maiores obtidos com outras fibras lignocelulósicas reforçando matrizes poliméricas [1ㅡ]

\section{CONCLUSÕES}

Compósitos fabricados com fibras contínuas e alinhadas de curauá reforçando matriz de poliéster apresentaram resistência à flexão em nível superior ao de outros compósitos poliméricos reforçados com fibras lignocelulósicas. 
A partir de 20\% em peso de fibra de curauá os compósitos atingem resistência próxima a 100 MPa o que é o triplo do melhor até agora obtido com fibras descontínuas e aleatórias.

As características compactas e alinhadas das fibrilas que formam cada fibra de curauá contribuem para maior aderência da resina poliéster, formando mini-compósitos que efetivamente participam na transferência dos esforços da matriz e, conseqüentemente, para o aumento da resistência do compósito.

\section{AGRADECIMENTOS}

O apoio a esta pesquisa concedido pelo CNPq, CAPES e FAPERJ na forma de recursos financeiros e/ou bolsas é motivo de agradecimento por parte dos autores deste trabalho que também agradecem a permissão do PEMM da COPPE/UFRJ para uso do MEV e a colaboração de Márcia Soares Sader e Felipe Sabatino Gonçalves Vieira

\section{REFERÊNCIAS}

[1] LEÃO, A.L., TAN, I.H., CRASCHI, J.C., "Curaua fiber - A tropical natural fibers from Amazon Potential and Application in Composites”, In: International Conference on Advanced Composites, pp. 557-564, Hurghada, Egito, 1998.

[2] FROLLINI, E., LEÃO, A.L., MATTOSO, L.H.S., Natural Polymers and Agrofibers Composites, pp. 257 - 272, Botucatu, Brasil, USP e UNESP, 2000.

[3] ERENO, D., "Fibra para Toda Obra”, Boletim Eletrônico Fapesp, n. 104, 2004. http://www.fapesp.br/agencia/boletim_dentro.php?data\%5Bid_materia_boletim\%5D=2621, Acesso em 10/02/2006.

[4] SABAA, M.W., "Mechanical properties of Curaua Fibers", Polymeric Degradation and Stability, v. 32, pp. $209-218,1991$.

[5] ARAUJO, C.R., PERLAZA, L.C, MOTHÉ, C.G., "Thermal Properties of Commercial and castor oil Polyurethane Composites with Curaua Fiber”, Natural Polymers and Composites, v. 4, pp. 547 550, 2002.

[6] ARAUjO, C.R., Cinética de Decomposição Térmica de Compósitos Poliméricos com Fibras de Curauá, Tese de D.Sc., EQ/UFRJ, Rio de Janeiro, RJ, Brasil, dezembro de 2003.

[7] MONTEIRO, S.N., DE DEUS, J.F., D’ALMEIDA, J.R.M., "Mechanical and Structural Characterization Of Curaua Fibers", In: Characterization of Minerals, Metals \& Materials - TMS Conference, pp. 18, San Antonio, USA, 2006.

[8] BLEDZKI, A.K., GASSAN, J., "Composites Reinforced with Cellulose-Based Fibres”, Progress in Polymer Science, v. 24, pp. 221-274, 1999.

[9] AGARWAL, B.D., BROUTMAN, L. J., Analysis and Performance of Fiber Composites, Nova York, John Wiley \& Sons, 1990.

[10] ASHBEE, K.H.G., Fundamental Principles of Fiber Reinforced Composites, Lancaster, Technomic Pub. Co. Inc., 1993.

[11] ASTM D-790 - Flexural Strength Testing of Plastics.

[12] MONTEIRO, S.N., DE DEUS, J.F., D’ALMEIDA, J.R.M., "Interfacial Strength of Curaua Fiber Reinforced Polyester Composites”, In: Jornadas SAM-CONAMET, pp. 1-6, Mar del Plata, Argentina, 2005.

[13] AQUINO, R.C.M.P., D’ALMEIDA, J.R.M., MONTEIRO, S.N., "Flexural Mechanical Porperties of of Piassava Fibers (Attalea funifera)-Resin Matrix Composites”, Journal of Materials Science Letters, v. 20, pp.1017-1019, 2001. 